\title{
Risk factors for postoperative complications following oral surgery
}

\author{
Hideo SHIGEISHI, Kouji OHTA, Masaaki TAKECHI
}

Department of Oral and Maxillofacial Surgery, Institute of Biomedical and Health Sciences, Hiroshima University, 1-2-3 Kasumi, Minami-ku, Hiroshima 7348553, Japan

Corresponding address: Hideo Shigeishi - Department of Oral and Maxillofacial Surgery, Institute of Biomedical and Health Sciences - Hiroshima University, 1-2-3 - Kasumi - Minami-ku - Hiroshima - 734-8553 - Japan - Phone: +81-82-257-5673, Fax: +81-82-257-5671 - e-mail: shige@hiroshima-u.ac.jp

Submitted: April 1, 2015 - Modification: June 1, 2015 - Accepted: June 3, 2015

\section{ABSTRACT}

\begin{abstract}
O bjective: The objective of this study was to clarify significant risk factors for postoperative complications in the oral cavity in patients who underwent oral surgery, excluding those with oral cancer. Material and Methods: This study reviewed the records of 324 patients who underwent mildly to moderately invasive oral surgery (e.g., impacted tooth extraction, cyst excision, fixation of mandibular and maxillary fractures, osteotomy, resection of a benign tumor, sinus lifting, bone grafting, removal of a sialolith, among others) under general anesthesia or intravenous sedation from 2012 to 2014 at the Department of Oral and Maxillofacial Reconstructive Surgery, Hiroshima University Hospital. Results: Univariate analysis showed a statistical relationship between postoperative complications (i.e., surgical site infection, anastomotic leak) and diabetes $(p=0.033)$, preoperative serum albumin level $(p=0.009)$, and operation duration $(p=0.0093)$. Furthermore, preoperative serum albumin level $(<4.0 \mathrm{~g} / \mathrm{dL}$ ) and operation time ( $\geq 120$ minutes) were found to be independent factors affecting postoperative complications in multiple logistic regression analysis results (odds ratio $3.82, p=0.0074$; odds ratio $2.83, p=0.0086$, respectively). Conclusion: Our results indicate that a low level of albumin in serum and prolonged operation duration are important risk factors for postoperative complications occurring in the oral cavity following oral surgery.
\end{abstract}

Keywords: Oral surgery. Postoperative complications. Blood serum albumin. Retrospective studies.

\section{INTRODUCTION}

The oral cavity harbors a large number of organisms, which can delay normal wound healing or cause infection in a surgical region. Indeed, surgical site infection and aspiration pneumonia are common complications following oral surgery 3,7 , with the former as an important cause of prolonged hospitalization and reduced patient quality of life. In head and neck cancer patients, the rate of surgical site infection ranges from $10 \%$ to $45 \%$, with previous chemotherapy, performance of a tracheotomy, free flap reconstruction, blood loss volume, and hypoalbuminemia noted as risk factors $5,6,8,10$. These findings suggest that severely invasive surgery (i.e., reconstructive surgery, flap surgery) and host nutritional status are predictive risk factors for head and neck cancer patients. In addition, a recent study showed that preoperative oral health care can reduce the risk of surgical site infection, indicating its importance to reduce postoperative complications ${ }^{12}$. However, few studies have been performed to determine predictive risk factors related to postoperative complications in patients who undergo oral surgery, except for oral cancer cases. In the present study, we focused on patients who underwent mildly to moderately invasive oral surgery in order to clarify significant risk factors related to postoperative complications in the oral cavity.

\section{METHODS}

This study reviewed the records of 324 patients (171 males, 153 females; with a mean age of 41.0 years, ranging from 5 to 84 years) who underwent 
surgical treatment in the operating room under general anesthesia or intravenous sedation at the Department of Oral and Maxillofacial Reconstructive Surgery, Hiroshima University Hospital, from 2012 and 2014. Subjects included in this study were those who underwent an operation for impacted tooth extraction, cyst excision, fixation of mandibular and maxillary fractures, jaw deformity (osteotomy), resection of a benign tumor, sinus lifting, bone grafting, removal of a sialolith, and others. Oral cancer patients who underwent primary resection, neck dissection, and reconstructive surgical procedures were excluded from the analysis. A summary of the surgical procedures performed is shown in Table 1.

Clinical data obtained included patient age, sex, medical history, blood loss volume, and operation duration. All operations were performed without using blood transfusion. As for the antibiotic drugs given, cephem antibiotics (e.g., cefdinir, cefmetazole, cefaclor, cefditoren pivoxil, flomoxef) and penicillin antibiotics (ampicillin) were administered in all cases for 3-7 days after the operation. Blood serum albumin was examined on the day before surgery. Hypoalbuminemia was defined as a serum albumin level below 4.0 $\mathrm{mg} / \mathrm{dl}$. We also investigated the occurrence of complications such as anastomotic leak and surgical site infection within 14 days after surgery. Surgical site infection was determined according to the method of Jonson, et $\mathrm{al}^{4}$ (1984) Fisher's exact test and a multivariate logistic regression model were used for statistical analysis, with $p$ values less than 0.05 regarded to be statistically significant. In addition, a Mann-Whitney $U$ test was utilized for nonparametric statistical analysis of serum albumin level, blood loss volume, and duration time. This retrospective study was approved by the Ethics Committee of Hiroshima University.

\section{RESULTS}

We investigated the relationship between clinical factors and postoperative complications (e.g., surgical site infection, anastomotic leak). A total of 36 cases had postoperative complications (overall rate of $11.1 \%$ ), of which four showed surgical site infection and 32 an anastomotic leak without a distinct infection sign. Then, we analyzed the correlation between postoperative complications and clinical factors including gender, age, diabetes, respiratory disorder, cardiovascular disease, preoperative serum albumin level, surgical procedure, blood loss volume, and operation time (Fisher's exact test) (Table 2). As for antibiotic drugs, there were no apparent effects of a specific antibiotic on postoperative complications (data not shown). On the other hand, the percentage of complications was increased in diabetic (33.3\%) as compared to non-diabetic $(10.3 \%)$ patients (Table 2), and also increased in patients with low level of serum albumin (29.2\%) as compared to those with a high level (9.7\%) (Table 2). In univariate analysis, we found a significant relationship between postoperative complications and diabetes $(p=0.033)$, preoperative serum albumin level $(p=0.009)$, and operation duration $(p<0.0093)$ (Table 2$)$. Next, those three variables were evaluated using a multiple logistic regression model. As shown in Table 3, preoperative serum albumin level $(<4.0 \mathrm{~g} / \mathrm{dL})$ and operation time ( $\geq 120$ minutes) were found to be independent risk factors affecting postoperative complications (odds ratio $3.82, p=0.0074$; odds ratio $2.83, p=0.0086$, respectively).

Among the continuous variables, preoperative serum albumin, blood loss volume, and operation duration were examined using a Mann-Whitney $U$ test for nonparametric statistical analysis. Preoperative serum albumin level was significantly lower in patients who developed a surgical site infection or anastomotic leak $(p=0.025)$. In

Table 1- Summary of surgical procedures performed in the present cases

\begin{tabular}{lc}
\hline \multicolumn{1}{c}{ Surgical procedures } & Patients (\%) \\
\hline Impacted tooth extraction & $107(33.0 \%)$ \\
\hline Excision of jaw bone cyst/radicular cyst & $94(29.0 \%)$ \\
\hline Fixation of mandibular/maxillary fracture & $41(12.7 \%)$ \\
Resection of benign tumor & $34(10.5 \%)$ \\
Jaw bone osteotomy for jaw deformity & $27(8.3 \%)$ \\
Sinus lifting & $9(2.8 \%)$ \\
Removal of sialolith & $5(1.5 \%)$ \\
Bone graft & $5(1.5 \%)$ \\
\hline Frenuloplasty & $1(0.31 \%)$ \\
Excision of sublingual gland and mucocele & $1(0.31 \%)$ \\
\hline
\end{tabular}


Table 2- Correlation between clinical characters and postoperative complications

\begin{tabular}{|c|c|c|c|}
\hline \multirow[b]{2}{*}{ Clinical characteristics } & \multicolumn{2}{|c|}{ Postoperative complications } & \multirow[b]{2}{*}{$p$ value } \\
\hline & $(-)$ & $(+)$ & \\
\hline \multicolumn{4}{|l|}{ Gender } \\
\hline Male (171) & $150(87.7 \%)$ & $21(12.3 \%)$ & 0.60 \\
\hline Female (153) & $138(90.2 \%)$ & $15(9.8 \%)$ & \\
\hline \multicolumn{4}{|l|}{ Age in years } \\
\hline$<60(253)$ & $226(89.3 \%)$ & $27(10.7 \%)$ & 0.67 \\
\hline$\geq 60(71)$ & $62(87.3 \%)$ & $9(12.7 \%)$ & \\
\hline \multicolumn{4}{|l|}{ Diabetes } \\
\hline$(-)(312)$ & $280(89.7 \%)$ & $32(10.3 \%)$ & $0.033^{*}$ \\
\hline$(+)(12)$ & $8(66.7 \%)$ & $4(33.3 \%)$ & \\
\hline \multicolumn{4}{|l|}{ Cardiovascular disease } \\
\hline$(-)(287)$ & $255(88.9 \%)$ & $32(11.1 \%)$ & 1.0 \\
\hline$(+)(37)$ & $33(89.2 \%)$ & $4(10.8 \%)$ & \\
\hline \multicolumn{4}{|l|}{ Respiratory disease } \\
\hline$(-)(315)$ & $280(88.9 \%)$ & $35(11.1 \%)$ & 1.0 \\
\hline$(+)(9)$ & $8(88.9 \%)$ & $1(11.1 \%)$ & \\
\hline \multicolumn{4}{|l|}{ Preoperative serum albumin } \\
\hline$<4.0 \mathrm{~g} / \mathrm{dL}(24)$ & $17(70.8 \%)$ & $7(29.2 \%)$ & $0.0099^{* *}$ \\
\hline$\geq 4.0 \mathrm{~g} / \mathrm{dL}(300)$ & $271(90.3 \%)$ & $29(9.7 \%)$ & \\
\hline \multicolumn{4}{|l|}{ Surgical procedure } \\
\hline Impacted tooth extraction (107) & $97(90.7 \%)$ & $10(9.3 \%)$ & 0.27 \\
\hline Excision of jaw bone cyst (94) & $87(92.6 \%)$ & $7(7.4 \%)$ & \\
\hline Fixation of fractures (41) & $34(82.9 \%)$ & $7(17.1 \%)$ & \\
\hline Resection of benign tumor (34) & $30(88.2 \%)$ & $4(11.8 \%)$ & \\
\hline Jaw bone osteotomy (27) & $24(88.9 \%)$ & $3(11.1 \%)$ & \\
\hline Sinus lifting (9) & $6(66.7 \%)$ & $3(33.3 \%)$ & \\
\hline Removal of sialolith (5) & $4(80.0 \%)$ & $1(20.0 \%)$ & \\
\hline Bone graft (5) & $4(80.0 \%)$ & $1(20.0 \%)$ & \\
\hline Frenuloplasty (1) & $1(100.0 \%)$ & $0(0.0 \%)$ & \\
\hline Excision of sublingual gland (1) & $1(100.0 \%)$ & $0(0.0 \%)$ & \\
\hline \multicolumn{4}{|l|}{ Blood loss } \\
\hline$<300 \mathrm{ml}(307)$ & $274(91.3 \%)$ & $33(10.7 \%)$ & 0.42 \\
\hline$\geq 300 \mathrm{ml}(17)$ & $14(82.4 \%)$ & $3(17.6 \%)$ & \\
\hline \multicolumn{4}{|l|}{ Operation time } \\
\hline$<120 \min (269)$ & $245(91.1 \%)$ & $24(8.9 \%)$ & $0.0093^{\star *}$ \\
\hline$\geq 120 \min (55)$ & $43(78.2 \%)$ & $12(21.8 \%)$ & \\
\hline
\end{tabular}

Fisher's exact test was used for statistical analysis. Statistical significances of $p<0.05$ and $p<0.01$ were indicated by * and ** respectively

addition, blood loss volume $(p=0.014)$ and operation duration $(p=0.0065)$ were significantly increased in patients with postoperative complications. These results suggest that low serum albumin, increased blood loss volume, and prolonged operation time are importantly associated with postoperative complications following oral surgery (Table 4).

\section{DISCUSSION}

Postoperative complications often result in prolonged hospital stay and reduced quality of life 
Table 3- Multivariate logistic regression analysis of predictive factors for postoperative complications

\begin{tabular}{lccc}
\hline \multicolumn{1}{c}{ Variables } & Odds ratio & 95\% confidence interval & p value \\
\hline Step 1 & & & \\
Diabetes $(+)$ & 3.72 & $0.97-14.3$ & 0.056 \\
Preoperative serum albumin $(<4.0 \mathrm{~g} / \mathrm{dL})$ & 3.35 & $1.22-9.2$ & 0.019 \\
Operation time $(\geq 120 \mathrm{~min})$ & 2.89 & $1.31-6.35$ & 0.0084 \\
Step 2 & & & \\
Preoperative serum albumin $(<4.0 \mathrm{~g} / \mathrm{dL})$ & 3.82 & $1.43-10.2$ & $0.0074^{\text {** }}$ \\
Operation time $(\geq 120 \mathrm{~min})$ & 2.83 & $1.30-6.17$ & $0.0086^{\text {** }}$ \\
\hline
\end{tabular}

A multivariate logistic regression model was used for statistical analysis. Statistical significances of $p<0.05$ and $p<0.01$ were indicated by * and ${ }^{* *}$ respectively

Table 4- Correlation between postoperative complications and serum albumin level, blood loss, or operation time

\begin{tabular}{lccc}
\hline & \multicolumn{2}{c}{ Postoperative complications } \\
\hline Variables & $(-)$ & $(+)$ & p value \\
Preoperative serum albumin $(\mathrm{g} / \mathrm{dL})$ & $4.53 \pm 0.39$ & $4.33 \pm 0.48$ & $0.025^{*}$ \\
Blood loss $(\mathrm{mL})$ & $65.6 \pm 142.5$ & $104.0 \pm 168.5$ & $0.014^{*}$ \\
Operation time $(\mathrm{min})$ & $84.5 \pm 71.0$ & $108.4 \pm 75.2$ & $0.0065^{* *}$ \\
\hline
\end{tabular}

Mann-Whitney $U$ test was utilized for nonparametric statistical analysis of serum albumin level, blood loss volume, and duration time. Statistical significances of $p<0.05$ and $p<0.01$ were indicated by * and ** respectively

in affected patients. Therefore, it is important to identify risk factors associated with postoperative complications and eliminate them as much as possible. In this study, we examined some clinical factors to determine their relationship with postoperative complications such as surgical site infection and anastomotic leak. Of 36 cases with complications, four had an apparent infection in the surgical region, whereas most $(n=32)$ had an anastomotic leak. In our patients, the administration of antibiotic prophylaxis may have reduced the opportunity for infection, resulting in the relatively small number of surgical site infection cases in the present cohort.

Albumin level, in particular, is thought to be a reliable marker of nutritional status in patients following surgery ${ }^{11}$, with a decreased level associated with rheumatoid disease, indicating it as a promising marker of inflammatory disease ${ }^{2}$. Thus, that level is also considered to be a significant and useful factor to assess malnutrition and inflammation in patients. In addition, serum albumin is thought to be a predictive nutritional parameter for patients with head and neck cancer ${ }^{14}$. In a previous study, preoperative albumin level in serum was identified as significant risk factor for surgical site infection in those who underwent primary resection, and head and neck reconstructive surgery ${ }^{6}$. The present findings also showed that decreased preoperative serum albumin was associated with postoperative complications in patients who underwent oral surgery. Together, these characteristics suggest that a low level of albumin in serum is a reliable factor to predict complications in patients undergoing oral surgery. Furthermore, it may be possible to reduce the risk of surgical complications by improving their nutritional status ${ }^{13}$.

Our results also showed that prolonged operation duration and increased blood loss volume were associated with postoperative complications in the evaluated patients. These clinical parameters are dependent on the severity of the surgical procedure and skill of the surgeon. Thus, severely invasive surgery and poor surgical skill may be associated with prolonged duration and a high volume of blood loss. Previous studies identified significant associations of operation time and blood loss with surgical infection in patients who underwent head and neck surgery ${ }^{6,8,12}$. In contrast, another report noted no significant relationships between those in head and neck cancer patients ${ }^{5}$. Even though it remains controversial whether operation time and blood loss are important risk factors for complications following oral surgery, it is important to minimize blood loss and reduce operation time by improving surgical techniques and therapeutic strategies.

Evidence has been presented showing that patients with diabetes are at high risk of slow healing and wound infection following an operation ${ }^{9}$. However, it remains undetermined whether those who undergo oral surgery are more likely to have 
a risk for surgical infection as compared to nondiabetic patients ${ }^{1}$. In some studies of head and neck cancer patients, no significant correlation between diabetes and surgical infection was found ${ }^{5,8,12}$. In the present study, univariate but not multivariate analysis showed a significant relationship between diabetes and postoperative complications. The diabetic patients who participated in our study were properly controlled by antidiabetic drug or insulin administrations during their hospital stay. Although long-term glycemic control may have an influence, the effect of diabetes on postoperative complications remains unclear. Further investigations are necessary to clarify the effect of diabetes on patients who undergo oral surgery.

Postoperative oral hygiene is considered necessary for patients who have difficulty with oral cleaning due to surgical damage, as microflora harbored in the oral cavity may be composed of pathogens related to surgical site infection. Recently, oral health care has become recognized as essential to decrease postoperative complications in head and neck cancer, as well as other patients with cancer $^{12,15}$. A few of the evaluated patients received professional oral health care prior to surgery, thus, we were unable to examine the effects of that on postoperative complications. However, we have observed that preoperative oral health care reduces the percentage of postoperative complications in head and neck cancer patients (unpublished data). We consider that professional oral health care can reduce the risk of postoperative complications in cases of oral surgery.

\section{CONCLUSION}

The present findings indicate that hypoalbuminemia and prolonged operation duration are significant risk factors for postoperative complications in patients who undergo oral surgery.

\section{Funding}

This work was supported by a Grant-in-aid (no. 23592963) from the Ministry of Education, Culture, Sports, and Technology of Japan.

\section{Ethical approval}

The study was approved by the Ethics Committee of Hiroshima University (approval no. 1103).

\section{REFERENCES}

1- Barasch A, Safford MM, Litaker MS, Gilbert GH. Risk factors for oral postoperative infection in patients with diabetes. Spec Care Dentist. 2008;28:159-66

2- Fanali G, di Masi A, Trezza V, Marino M, Fasano M, Ascenzi P. Human serum albumin: from bench to bedside. Mol Aspects Med. 2012;33:209-90.

3- Grandis JR, Snyderman CH, Johnson JT, Yu VL, D'Amico F. Postoperative wound infection. A poor prognostic sign for patients with head and neck cancer. Cancer. 1992;15:2166-70.

4- Johnson JT, Myers EN, Thearle PB, Sigler BA, Schramm VL Jr. Antimicrobial prophylaxis for contaminated head and neck surgery. Laryngoscope. 1984;94:46-51.

5- Kamizono K, Sakuraba M, Nagamatsu S, Miyamoto S, Hayashi R. Statistical analysis of surgical site infection after head and neck reconstructive surgery. Ann Surg Oncol. 2014;21:1700-5.

6- Liu SA, Wong YK, Poon CK, Wang CC, Wang CP, Tung KC. Risk factors for wound infection after surgery in primary oral cavity cancer patients. Laryngoscope. 2007;117:166-71.

7- McCulloch TM, Jensen NF, Girod DA, Tsue TT, Weymuller EA Jr. Risk factors for pulmonary complications in the postoperative head and neck surgery patient. Head Neck. 1997;19:372-7.

8- Ogihara H, Takeuchi K, Majima Y. Risk factors of postoperative infection in head and neck surgery. Auris Nasus Larynx. 2009;36:457-60.

9- Peleg AY, Weerarathna T, McCarthy JS, Davis TM. Common infections in diabetes: pathogenesis, management and relationship to glycaemic control. Diabetes Metab Res Rev. 2007;23:3-13.

10- Penel N, Fournier C, Lefebvre D, Lefebvre JL. Multivariate analysis of risk factors for wound infection in head and neck squamous cell carcinoma surgery with opening of mucosa. Study of 260 surgical procedures. Oral Oncol. 2005;41:294-303.

11- Santos NS, Draibe SA, Kamimura MA, Canziani ME, Cendoroglo $M$, Gabriel Júnior $A$, et al. Is serum albumin a marker of nutritional status in hemodialysis patients without evidence of inflammation? Artif Organs. 2003;27:681-6.

12- Sato J, Goto J, Harahashi A, Murata T, Hata H, Yamazaki Y, et al. Oral health care reduces the risk of postoperative surgical site infection in inpatients with oral squamous cell carcinoma. Support Care Cancer. 2011;19:409-16.

13- Snyderman CH, Kachman K, Molseed L, Wagner R, D'Amico F, Bumpous J, et al. Reduced postoperative infections with an immune-enhancing nutritional supplement. Laryngoscope. 1999;109:915-21.

14- Varkey P, Tang WR, Tan NC. Nutrition in head and neck cancer patients. Semin Plast Surg. 2010;24:325-30.

15- Yoneda S, Imai S, Hanada N, Yamazaki T, Senpuku H, Ota Y, et al. Effects of oral care on development of oral mucositis and microorganisms in patients with esophageal cancer. Jpn J Infect Dis. $2007 ; 60: 23-8$. 